\title{
Nerve-preserving Cytoreductive Surgery and Hyperthermic Intraperitoneal Chemotherapy for Advanced Primary Caecal Cancer Invading Pelvic Side-wall: Report of a Case With Right Leg Limping
}

\author{
Ethem Unal ${ }^{1}$, Abdullah Yildiz ${ }^{2}$ and Sema Yuksekdag ${ }^{2}$ \\ ${ }^{1}$ Department of Surgical Oncology, Health Sciences University of Istanbul, Istanbul, Turkey \\ ${ }^{2}$ Department of General Surgery, Health Sciences University of Istanbul, Istanbul, Turkey
}

\begin{abstract}
Radical surgery to achieve optimal cytoreduction in locally advanced caecal cancer may dictate femoral or sciatic nerve resection, especially in cases with pelvic side-wall involvement. In such a situation, gait disturbance is inevitable. A 61-year male with a new-onset right leg limping due to locally advanced right colon carcinoma underwent cytoreductive surgery (CRS) with partial resection of right pelvic side- wall, and hyperthermic intraperitoneal chemotherapy (HIPEC). During the operation, we aimed to preserve both the sciatic and femoral nerves to prevent further deterioration of his right leg limping postoperatively. However, we did not compromise the principles of radical surgery and all tumoral implants around femoral vessels and nerves were removed. Completeness of cytoreduction score was zero (CCO). The resection of all macroscopic disease is the main goal of CRS, but as seen in our case with tumor-related walking difficulty, nerve-sparing CRS and HIPEC may prevent further deterioration of the situation.
\end{abstract}

Key Words: Cytoreductive surgery, Hyperthermic intraperitoneal chemotherapy, Sciatic nerve, Femoral nerve, Caecal cancer.

How to cite this article: Unal E, Yildiz A, Yuksekdag S. Nerve-preserving Cytoreductive Surgery and Hyperthermic Intraperitoneal Chemotherapy for Advanced Primary Caecal Cancer Invading Pelvic Side-wall: Report of a Case With Right Leg Limping. J Coll Physicians Surg Pak 2021; 31(07):858-860.

\section{INTRODUCTION}

In recent years, the management of both primary and metastatic peritoneal malignancies with cytoreductive surgery (CRS) and hyperthermic intraperitoneal chemotherapy (HIPEC) has gained great popularity, and has resulted in a radical change from simple palliation to possible cure. ${ }^{1}$ Pelvic exenteration to achieve optimal cytoreduction may dictate urinary and intestinal reconstructions and pelvic side - wall resection. Even today, advanced pelvic tumors such as those invading or compressing the sciatic or femoral nerves are considered inoperable. However, some recent studies have reported acceptable functional outcomes after radical surgery with en-bloc sciatic and/or femoral nerve resection. ${ }^{2,3}$ In these studies, physical quality of life (QoL) has been shown to be impaired after surgery, but returns to baseline by 12 months in some cases. ${ }^{4}$

Correspondence to: Dr. Ethem Unal, Department of Surgical Oncology, Health Sciences University of Istanbul, Istanbul, Turkey

E-mail:drethemunal@gmail.com

Received: September 30, 2019; Revised: November 15, 2019; Accepted: November 27, 2019

DOI: https://doi.org/10.29271/jcpsp.2021.07.858
We, herein, present a case of a 61-year male with tumor-induced right leg limping, suffering from advanced right colon carcinoma, who underwent CRS with partial resection of right pelvic side wall- sparing both sciatic and femoral nerves and HIPEC.

\section{CASE REPORT}

A 61-year male with advanced caecal tumor, causing limping due to pelvic side-wall invasion, was admitted and prepared for CRS and HIPEC. Preoperative computerised tomography (CT) showed a locally advanced tumor; and colonoscopic biopsy was reported as adenocarcinoma. In addition to these findings, positron emission tomography (PET) scan revealed pelvic sidewall invasion. Preoperative biochemistry parameters were normal except a mild anemia, moderately elevated tumor markers and increased glucose levels. The patient had difficulty in walking for three weeks, and his comorbidities were hypertension and uncontrolled diabetes mellitus. After controlling the diabetes mellitus, operation was performed. Abdominal exploration revealed conglomerate loops of ileocaecal and caecal segments fixed to the abdominal wall and pelvis. Peritoneal carcinomatosis index (PCI) was 12. Right rectus abdominis and ileopsoas muscles were partially resected after righthemicolectomy. Dissection revealed the involvement of the right iliac crest along with femoral and sciatic nerves. Sparing these 
anatomical structures, the tumoral implants around femoral vessels and nerves were removed. Following Kocher manoeuvre, hepatoduodenal ligament was visualised and no implants were seen. The hydropic gallbladder was resected. There was no visible tumor remaining behind, so the completeness of cytoreduction (CC) score was 0 (Figure 1). After HIPEC procedure using oxaliplatin, $460 \mathrm{mg} / \mathrm{m}^{2}$ at $41^{\circ} \mathrm{C}$ for 30 minutes, with the closed abdomen technique and 2 inflow and 2 outflow catheters (Figure 2), ileotransversostomy was performed with a stapler; and anastomosis was strengthened with interrupted vicryl sutures. Postoperative course was unremarkable except a prolonged ileus, and patient was discharged on the $12^{\text {th }}$ postoperative day. The patient is alive and doing well at the $11^{\text {th }}$ postoperative month, without any gait disturbance. He received adjuvant chemotherapy and his last PET scan showed no recurrence. His walking improved significantly following surgery, without further need of physical therapy or any other intervention.

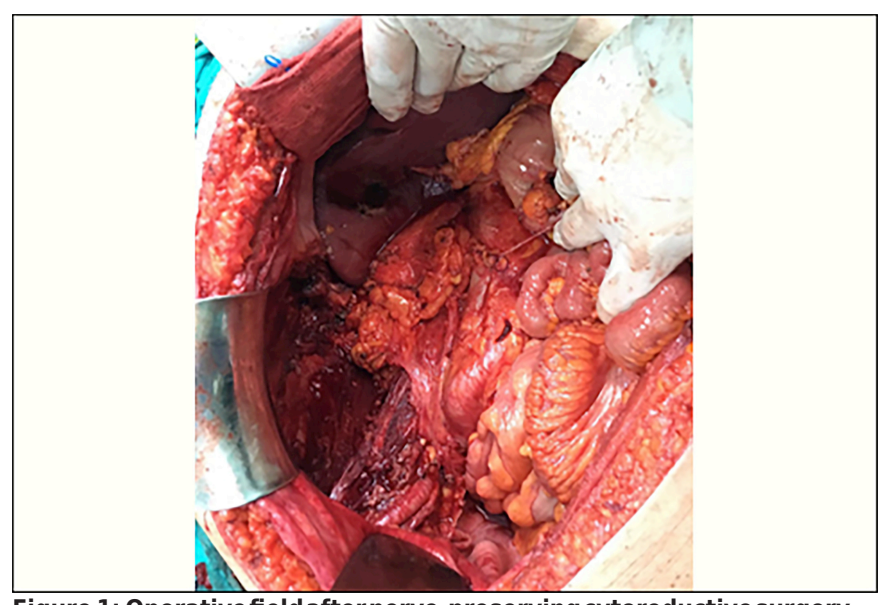

Figure 1: Operative field after nerve-preserving cytoreductive surgery.

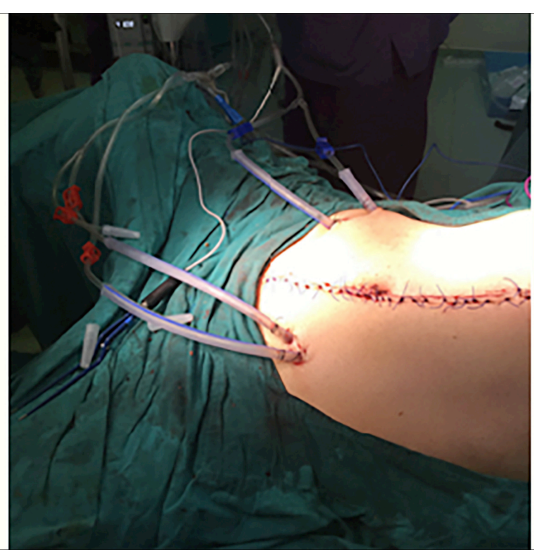

Figure 2: HIPEC procedure (using oxaliplatin for $\mathbf{3 0}$ minutes with the closed abdomen technique and 2 inflow and 2 outflow catheters).

\section{DISCUSSION}

Recently, there has been a world-wide trend towards more radical CRS for patients with advanced gynecologic and gastrointestinal carcinomas. ${ }^{5,6}$ Traditional simple pelvic resections are preferred less frequently, and CRS with intense local chemotherapy has gained a considerable popularity.
In our case with a locally advanced caecal tumor causing femoral and sciatic nerve compression, we preferred to preserve both the sciatic and femoral nerves to prevent further deterioration of his right leg limping postoperatively, while not compromising the principles of radical surgery to perform a ccoresection.

Sciatic nerve, the largest nerve in the body, contains fibers from both the anterior and posterior rami of the lumbosacral (LS) plexus. It is derived from spinal nerves L4 to S3, and innervates nearly the whole of the skin of lower extremity and muscles of the thigh, leg and foot. ${ }^{4}$ Femoral nerve originates from $L 2$ to $L 4$ roots to form the lunar plexus, passes along the lateral border of psoas muscle and enters the thigh., It innervates pectineous and sartorius muscles, and carries sensations from the medial surface of the thigh. ${ }^{3}$ It is also responsible for the motor function of quadriceps muscle and the sensory innervation of the medial side of the lower extremity from the knee to the ankle. Injury to these nerves causes both motor and sensory deficits, and results in gait disturbances.

Although some recent studies have suggested that en-bloc sciatic and femoral nerve resections may be performed during extended radical pelvic resections with acceptable morbidity and survival, the same studies nevertheless report a marked impairment of the physical QoL right after surgery. ${ }^{4}$ As seen in our case, the existence of preoperative gait disturbance may compel the surgeon to perform nerve-sparing surgery without breaking the standards of CRS. Furthermore, HIPEC procedure will also secure $\mathrm{CCO}$ resection. In our opinion, the challenging tumors, which are considered inoperable due to various vital structural involvements, might be resected in selected cases preserving both oncological principles and organ functionality, such as in our case in which both tumor-free margins and recovery of symptoms related to nerve compressions were achieved.

\section{ACKNOWLEDGEMENT:}

We would like to express our special appreciation, and thanks to Dr. Omer Faruk Ozkan for taking part in such a complicated operation, especially in the resection part of the pelvic sidewall metastasis.

\section{PATIENT'S CONSENT:}

Written informed consent was obtained from the patient to publish the data concerning the present case.

\section{CONFLICT OF INTEREST:}

The authors declared no conflict of interest.

\section{AUTHORS' CONTRIBUTION:}

EU: Surgical procedure, writing the manuscript and proofreading.

AY, EU: Data analysis and interpretation of the surgical outcome.

SY: Design of the study, data collection and literature review. 


\section{REFERENCES}

1. Baratti D, Kusamura S, Laterza B, Balestra MR, Deraco M. Early and long-term postoperative management following cytoreductive surgery and hyperthermic intraperitoneal chemotherapy. World J Gastrointest Oncol 2010; 2(1): 36-43. doi: 10.4251/wjgo.v2.i1.36.

2. Verwaal VJ, Ruth S, Bree E, Sloothen GW, Tinteren H, Boot $\mathrm{H}$, et al. Randomised trial of cytoreduction and hyperthermic intraperitoneal chemotherapy versus systemic chemotherapy and palliative surgery in patients with peritoneal carcinomatosis of colorectal cancer. J Clin Oncol 2003; 21(20):3737-43. doi: 10.1200/JCO.2003. 04.187.

3. Solomon MJ, Brown KG, Koh CE, Lee P, Austin KK, Masya L. Lateral pelvic compartment excision during pelvic exenteration. $B r$ J Surg 2015; 102(13):1710-7. doi: 10.1002/bjs.9915.
4. Franko J, Shi Q, Goldman CD, Pockaj BA, Nelson GD, Goldberg RM, et al. Treatment of colorectal peritoneal carcinomatosis with systemic chemotherapy: A pooled analysis of north central cancer treatment group phase III trials N9741 and N9841. J Clin Oncol 2012; 30(3):263-7. doi: 10.1200/JC0.2011.37.1039.

5. Verwaal VJ, van Ruth S, de Bree E, van Sloothen GW, van Tinteren $\mathrm{H}$, Boot $\mathrm{H}$, et al. Randomised trial of cytoreduction and hyperthermic intraperitoneal chemotherapy versus systemic chemotherapy and palliative surgery in patients with peritoneal carcinomatosis of colorectal cancer. J Clin Oncol 2003; 21(20):3737-43. doi: 10.1200/JCO.2003. 04.187.

6. Sinno AK, Li X, Thompson RE, Tanner EJ, Levinson KL, Stone $\mathrm{RL}$, et al. Trends and factors associated with radical cytoreductive surgery in the United States: A case for centralised care. Gynecol Oncol 2017; 145(3):493-9. doi: 10.1016/j.ygyno.2017.03.020. 